\title{
Omental Application in the Treatment of Spinal Cord Injuries: A Controlled Study is Warranted
}

Harry S. Goldsmith*

Department of Neurosurgery, Clinical Professor of Neurosurgery, University of California, Davis, USA

\begin{abstract}
Over the past half century there have been few therapeutic breakthroughs for the treatment of spinal cord injuries. It has been previously shown that following a Spinal Cord Injury $(\mathrm{SCl})$, scar tissue develops at the site of injury through which axons are unable to penetrate to make appropriate neurological connections into the distal spinal cord. Over the past few years it has been reported that placing the omentum directly on a traumatized spinal cord can result in a favorable clinical result. The omentum has been shown to limit the development of scar tissue following $\mathrm{SCl}$, which strongly suggests that omental transposition to an $\mathrm{SCl}$ now appears warranted to justify a carefully controlled evaluation of the benefits of omental application to an SCl.

Spinal cord injuries are devastating events. Unfortunately, in spite of improved medical and surgical treatment of the condition, there has not been a significant improvement in the neurological results of these injuries during the past half century. The purpose of this paper is to suggest a surgical technique that might increase functional improvement in the future treatment of spinal cord injuries (SCI). A new approach to the problem seems warranted.

Over a hundred years ago, Ramon y Cajal, the famous neuro-histologist, stated that axons are unable to connect to neural structures distal to an $\mathrm{SCl}$ based on his observations that axons cannot penetrate through scar tissue which routinely develops at the site of an SCl [1]. A half century later, Dr. L.H. Freeman, confirmed Cajal's observations by showing that axons had the inherent capacity to grow distally within an injured spinal cord (SC), but their progress routinely encountered a scar barrier in which axons "simply form neuromata at the injury site" [2]. Based on these observations, if neuro-protection within an $\mathrm{SCl}$ is to be addressed, laboratory and clinical efforts should be increasingly aimed at preventing the development of scar tissue in an acute $\mathrm{SCl}$ and decreasing the scar barrier present in a chronic $\mathrm{SCl}$.
\end{abstract}

\section{Pathophysiology of an SC Injury}

It is important to understand the pathophysiological events that take place following an SCI in which there is deposition of edema and blood that routinely occurs rapidly after injury within the SC. This is the result of leakage of edema and blood through the porous endothelial lining of damaged capillaries located mainly in the central grey matter of the SC. The edema fluid is rich in plasma protein which has a high osmotic pressure that attracts an increasing amount of edema fluid. As edema and blood accumulates in the injured area, it causes an extensive physical swelling of the spinal cord which is contained within its nonyielding dura mater covering and the surrounding bony vertebral column, a condition that causes an increased interstitial pressure at the site of the SCI.

As the edema expansion intensifies, the interstitial pressure within the spinal cord continues to increase, causing compression of veins in the area. This action results in an elevated venous pressure which further enhances the capillary extrusion of edema fluid and blood elements from the porous blood vessels at the injury site. Under normal conditions, extracellular edema fluid in the spinal cord drains into perivascular spaces with eventual drainage into cerebrospinal fluid. This flow pattern is essential for edema fluid elimination since there are no lymphatics in the spinal cord. As the volume of edema accumulates in an injured SC, there is eventual blockage of the central canal, subarachnoid and subdural spaces so that the normal edema drainage system within the spinal cord is compromised.

Compensatory fluid mechanics develop within the spinal cord following injury in the attempt to decrease expanding edema accumulation by displacing edema fluid up and down the spinal cord [3]. This longitudinal fluid movement, however, cannot compensate for the increasing edema volume that develops in the injury area. As edema accumulation increases, it causes the interstitial pressure at the SC injury site to become excessive, causing capillary compression which eventually can diminish capillary perfusion to the point of total vascular occlusion. When this occurs, there is irreversible damage to neural tissue unless circulation can be restored within hours of injury.

The blood-spinal cord barrier normally prevents leakage of blood into an uninjured spinal cord. Following a spinal cord injury, however, the blood-spinal cord barrier is broken and blood enters the spinal cord. It becomes critical at this time to employ a technique to eliminate, or at least lessen, the deleterious effect of the free blood in the SC since blood is known to be injurious to neural tissue.

Blood which leaks from porous SC blood vessels after injury incorporates fibrinogen which is a normal component of the blood mixture. Fibrinogen is a soluble plasma protein synthesized in the liver that is converted into fibrin in the presence of blood coagulation. The key to eliminating spinal cord scarring may well rest with the absorption of fibrinogen. The evidence for this is that fibrinogen is deposited in the spinal cord following injury, which in turn inhibits

*Corresponding author: Harry S. Goldsmith, Department of Neurosurgery, Clinical Professor of Neurosurgery, University of California, Davis, USA, Tel: 775-749-5801; Fax: 775-749-5861; E-mail: Hlgldsmith@aol.com

Received March 08, 2012; Accepted April 04, 2012; Published April 10, 2012

Citation: Goldsmith HS (2012) Omental Application in the Treatment of Spinal Cord Injuries: A Controlled Study is Warranted. J Trauma Treat 1:127. doi:10.4172/21671222.1000127

Copyright: ( 2012 Goldsmith HS. This is an open-access article distributed under the terms of the Creative Commons Attribution License, which permits unrestricted use, distribution, and reproduction in any medium, provided the original author and source are credited. 
neurite outgrowth by triggering an inhibitory signal transduction pathway to neurons. This inhibition of neurite outgrowth by the presence of fibrinogen in the SC occurs as early as one day after injury, peaks at seven days, and decreases in the following weeks [4]. These activities indicate the importance of absorbing fibrinogen as early after injury as possible since the neurite inhibition by fibrinogen may begin the process of spinal cord scarring. Absorbing fibrinogen in the blood of an SC may eventually prove to be crucial to limiting scar formation within the injured SC through which axons cannot penetrate. The question is how blood with its fibrinogen component can be absorbed in the SC following injury.

\section{Evidence for Omental Absorption}

If blood can be considered important in the production of scar tissue in the SC following injury, the absorption of blood shortly after injury appears critical. An intact vascularized omental pedicle when placed directly on a traumatized spinal cord has this ability to absorb edema fluid and blood [5]. Apparently, the placement of an omental pedicle directly on an underlying injured spinal cord results in a dynamic equilibrium between the edema and blood which is present in the injured SC and the absorption of this material by the overlying omental pedicle. The absorptive capability of the omentum was first observed in the absorption of edema in lymphedematous arms of breast cancer patients following a radical mastectomy [6]. Subsequent laboratory studies showed that the omentum could absorb one-third of the entire cerebro-spinal fluid reservoir [7]. A most impressive demonstration to clearly show the enormous absorptive capability of the omentum is to place an intact omental pedicle graft from an animal into a beaker filled with saline and methylene blue dye. Within 30 seconds, the dye appears in lymphatics within the omentum (Figure 1). The reason for this absorptive speed is apparently due to the open ends of lymphatic vessels which allow fluid and dye to rapidly enter the lymphatic channels.

\section{Experimental Evidence of Omental Protection Following an SCI}

It has been shown that placing an intact omental pedicle graft on an acutely injured spinal cord in animals can lead to improved Somatosensory-Evoked Potential (SEP) activity and neurological function [8,9]. Cats were subjected to a 400-gram/centimeter weight drop onto their exposed spinal cord. An omental pedicle graft was placed directly onto the SC injury site in the experimental group. In the control group, however, the weight drop SC injury was created without placement of the omentum onto their SC injury site. All animals were sacrificed 30 days after injury. Animals in the control group without omental protection showed persistent edema fluid and scarring at the injury site (Figure 2A). The experimental group with omental protection of their SC showed the SC to be mainly spared of post-injury results (Figure 2B).

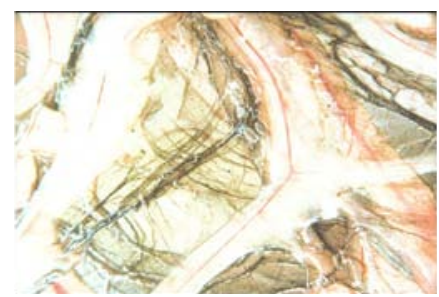

Figure 1: Blue dye in lymphatics after 30 second immersion in an India ink solution.

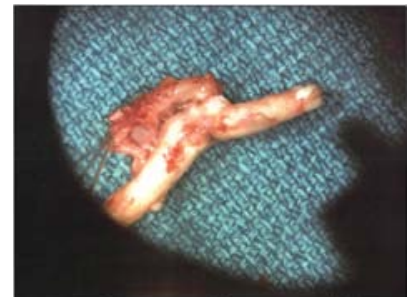

Figure 2A: Scar formation and edema remain at 30 days after injury.

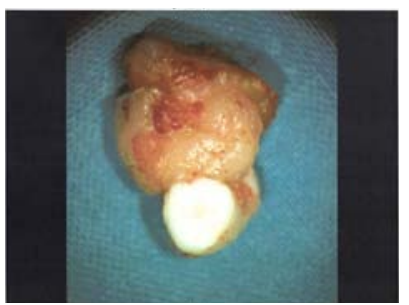

Figure 2B: No scar formation or edema noted at omental-spinal cord interface 30 days after injury.

\section{Omental Transposition Technique}

The surgical procedure of omental transposition to an injured spinal cord is not surgically difficult and has been previously described [10]. The procedure involves separation of the omental attachments to the transverse colon and to the proximal attachments to the greater curvature of the stomach, leaving the right gastroepiploic vessels intact within the omental apron. After these maneuvers, the omental apron has its blood supply coming entirely from the right gastric and right gastroepiploic vessels. The omental apron is then surgically tailored to create a long omental pedicle with its arterial and venous connections remaining intact. The omentum is then brought subcutaneously to the injured area of the spinal cord. A laminectomy is performed and the dura opened over the edematous site after which the omentum is laid directly on the spinal cord. The omentum is then sutured carefully to the cut edges of the dura.

\section{Clinical Approach to Treating an SCI}

\section{Acute spinal cord injuries}

In the acute period following an SCI in animals, omental application onto the injured SC demonstrated that the omentum could absorb edema and blood which lessened scar development at the injury site [11]. This strongly suggested that omental placement on the SC should be carried out as early after SCI as is clinically warranted since the sooner the fibrinogen present in blood can be absorbed at the SC injury site, the less chance for the development of scar which can begin to develop within days following injury. In acute SCI, the early absorption of blood from within the injured SC appears critical.

\section{Chronic Spinal Cord Injuries}

In acute SC injuries, the ideal treatment is to decrease the potential production of scar tissue by the early absorption of blood with its fibrinogen concentration. With a chronic spinal cord injury, however, scar tissue is already present within the $\mathrm{SC}$ which forms the barrier to post-injury penetration of axons. The treatment goal of a chronic SCI is to eliminate the scar tissue. The amount of scar tissue to be surgically removed may vary since in a chronic spinal cord injury the longitudinal 
length of the developed scar may be in direct proportion to the force of impact inflicted on the SC at the time of injury. All that might be necessary in a minor SC injury is the removal of a small amount of scar tissue, whereas in a severe SCI, a piece of the SC itself with its incorporation of scar tissue might require the removal of a portion of the SC. The challenge with a chronic SCI is to excise the scar tissue incorporated in the SC through which axons cannot penetrate.

A method has been developed to successfully replace the defect in the SC after total excision of an SC scar. It has been shown that following transection of the SC in cats, a $3 \mathrm{~cm}$ gap results in the spinal cord between the proximal and distal spinal cord stumps. This defect was corrected by constructing an omental-collagen bridge that connected the stumps of the transected SC (Figure 3). This surgical procedure in animals allowed axons to pass through the omental-collagen preparation (Figure 4A and Figure 4B) resulting in neurological improvement $[12,13]$. The progression of axons into the distal spinal cord was clearly demonstrated by retrograde dye studies which showed that distal synaptic connections had occurred [14-17].

The reason for the success of the SC omental-collagen bridging project was apparently the limited presence of blood with its fibrinogen component in the omental-collagen SC preparation. There is also the possibility that increased blood flow originating from the omentum into the omental-collagen bridge may have also played some role in

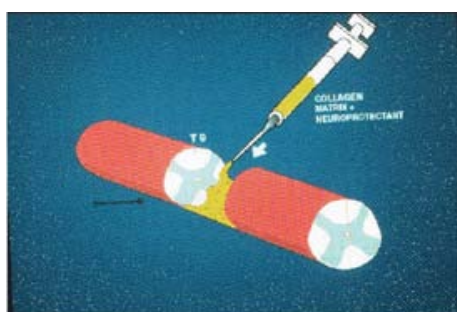

Figure 3: Collagen being inserted in gap connecting proximal and distal spina cord stumps. Omentum coverage will follow.

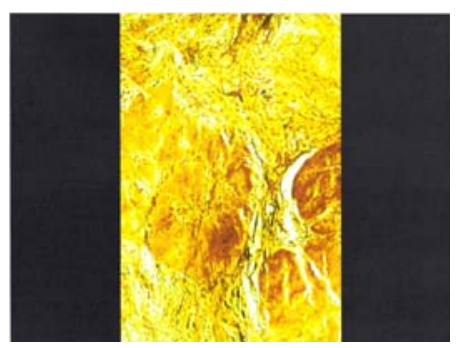

Figure 4A: Axons seen growing through center of omental-collagen bridge.

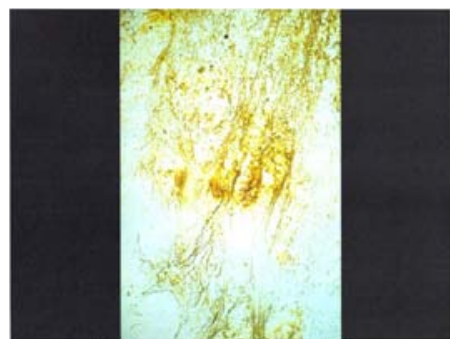

Figure 4B: Axons seen exiting distal end of omental-collagen bridge. Collagen is in process of biodegrading. the success of the operation. This possibility is based on experiments which showed that when the omentum is placed on an SCI site, the SC blood flow is increased by a mean of $58 \%$ as compared to a lower SC blood flow in control animals who lacked omental application to their injured SC [14]. In addition, when the omentum was placed on a totally transected SC which had been reconstructed by an omentalcollagen bridge, there was a 3:1 increase in blood vessel density counts penetrating into the omental-collagen bridge as compared to controls [18].

The successful omental-collagen bridging experiments in animals raised the question as to whether surgically removing SC scar tissue in chronic SCI patients could also result in comparable neurological improvement as had been observed in animals. This question was answered in one well-studied patient who had sustained a chronic spinal cord injury several years earlier.

\section{Case Report}

The patient was a 24-year-old woman who sustained a high-speed skiing accident $3^{1 / 4}$ years earlier. This injury resulted in a dislocation fracture at T-6-T-7 with fractures of the vertebral arches at T- 6 on the left and T-7 on the right. The most significant problem with her injury was a complete anatomical transection of her spinal cord at the T-6-T-7 level as reported by magnetic resonance imaging (MRI) (Figure 5). Over the next years following her injury, the patient retained complete motor loss at the T-6-T-7 level with an associated sensory loss at that level.

Thirty-nine months after her SCI the patient underwent reconstruction of her spinal cord using the same operative procedure that had been successfully used in cats; namely, bridging the transection gap between the divided SC cord stumps by the omental-collagen technique. This was done by totally removing her extensive SC scar which measured 1.6 inches $(4 \mathrm{~cm})$ in length (Figure 6). After removing the scar (Figure 7), 4cc. of collagen was inserted into the large SC (Figure 8) defect followed by placement of an omental pedicle directly on the underlying collagen (Figure 9).

Three months following surgery, the patient began rehabilitation. At 6 months she claimed she could actively move her legs on command. One year after surgery, she began to have trunk and abdominal wall strength (Figure 10). A follow-up MRI of her SC at this time showed a large soft tissue mass located between the proximal and distal spinal cord stumps (Figure 11). Subsequent MRIs taken over the next several years demonstrated the progressive development of a longitudinal extension of the soft tissue mass seen in the earlier MRI in association with further improvement in her neurological condition. Her neurological changes and MRI findings suggested that regenerating axons had passed through the previously noted soft tissue mass and had made distal connections with neural tissue below the SC transection site.

By four years after surgery, the patient had progressed to where she could walk for extensive distances but continued to require a walker since she lacked balance and was unstable without her walker (Figure 12). The patient's last MRI at six years after surgery showed the development of a longitudinal structure that connected the previously divided proximal and distal spinal cord stumps. Since collagen is biodegradable, it would appear that the longitudinal connection that had developed between the proximal and distal SC stumps on MRI must have incorporated neural structures (Figure 13). The ability of axons to penetrate through her SC omental-collagen bridge was theorized to 


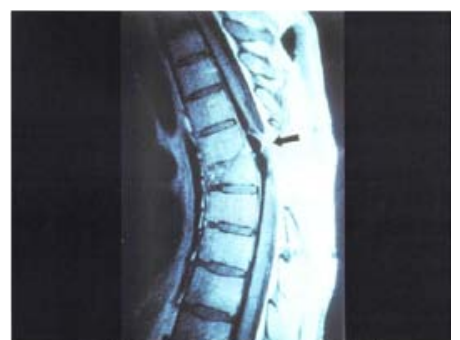

Figure 5: Arrow points to radiologically reported total anatomical spinal cord transection.

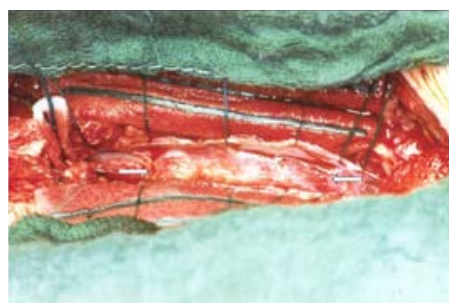

Figure 6: Arrows depict spinal cord scar between T6-T8.

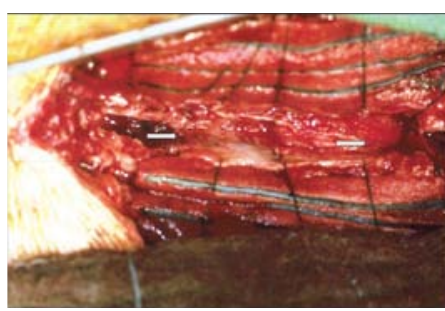

Figure 7: Arrows note absence of spinal cord between T6-T8.

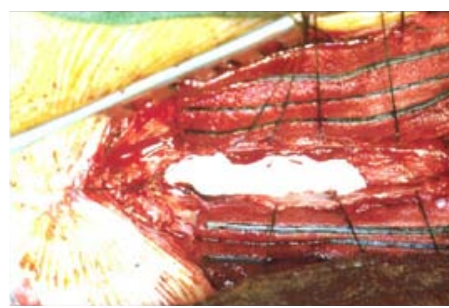

Figure 8: Photo showing collagen filling extensive spinal cord defect between T6- T8.

have occurred because of the absence of blood and fibrinogen which was not present at her previously constructed omental-collagen bridge.

\section{Discussion}

Over the past years there has been a host of technologies in the attempt to produce neuro-protection for SCI patients. Each decade promotes a major interest in this search for neuro-protection. Several decades ago there was enthusiasm for laminectomy and myelotomy based on the idea that these measures would lower the high interstitial pressure within an injured spinal cord resulting in a decrease in the swelling of the SC. The decreasing interstitial pressure in the SC was expected to lessen direct pressure on SC blood vessels which would allow for an increase in blood flow into the injured SC area, adequate blood supply being critical during any period of potential SC healing.
Interest in the use of laminectomy and myelotomy disappeared when it was shown to have little beneficial effects on SCI patients.

A subsequent method in the attempt to improve neurological function following SCI was the administration of the steroid, methylprednisolone, shortly following an SCI. This form of treatment became so popular in the United States that failure to use the drug in an SCI patient was considered medical malpractice and could result in a lawsuit against the doctor for not administering the drug. This form of treatment almost universally has lost favor over the past years.

More recent enthusiasm in the therapeutic treatment of an SC injury has been the use of stem cells in the hope that these cells could improve the neurological results following an SCI. At the recent Eighth International Symposium on Experimental Spinal Cord Repair and Regeneration (Italy, May 9-14, 2011), it was the conclusion of some researchers at this meeting that there is decreasing interest in the use of stem cells for an SCI. The reason for this is that definitive laboratory and clinical activity have not lived up to the considered potential of stem cells in treating an SCI. Stem cells are known to have many functions such as the replacement of lost neural tissue and angiogenic activity, but stem cells have no ability to absorb fibrinogen in blood during

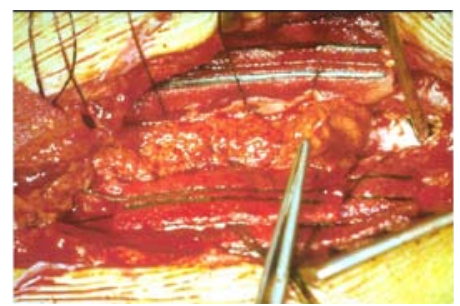

Figure 9: Photo showing pedicled omentum overlying collagen bridge.

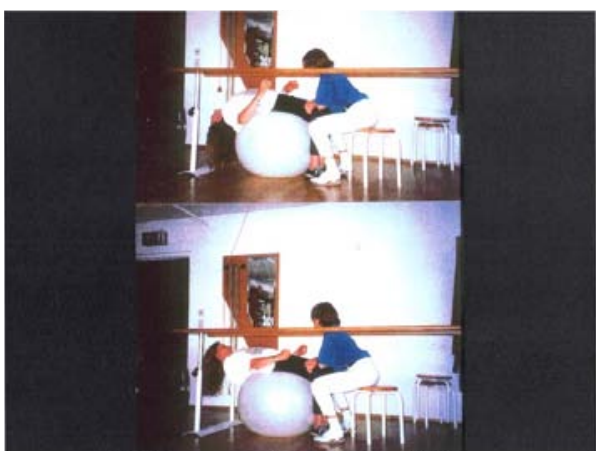

Figure 10: Marked post-operative strength of abdominal muscles that were paralyzed pre-operatively.

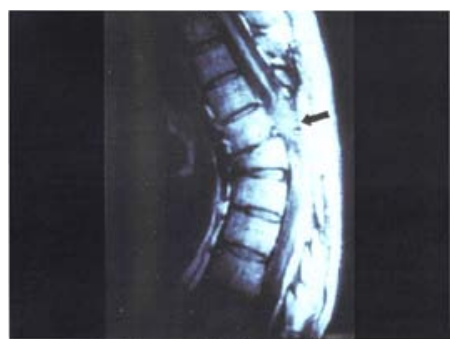

Figure 11: MRI of spinal cord one year after spinal cord reconstruction. Arrow points to soft tissue mass at T6-T8 


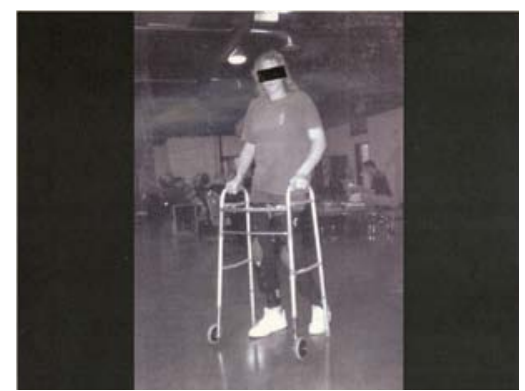

Figure 12: Photo showing patient using a walker

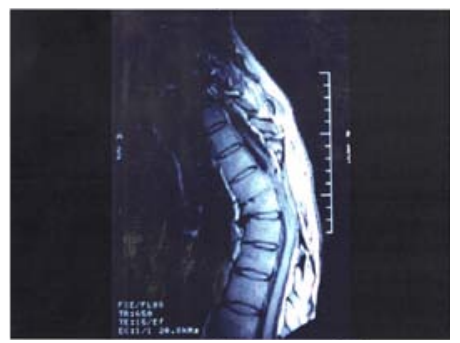

Figure 13: MRI at 6 years after surgery showing connection between proximal and distal segments of previously divided spinal cord.

the acute stage of an SCI and have no apparent effect in dismantling the scar barrier present in a chronic SCI. Without these two critical biological activities, it is understandable to question whether stem cells will ultimately demonstrate improvement in functional results following an SCI, especially in a severe SCI in which there is extensive scar tissue development.

A perceived decrease in enthusiasm in the use of stem cells for SC injuries has recently led to an increase in interest in the use of Spinal Cord Cooling (SCC) in treating an SCI $[19,20]$. Spinal cord cooling appears to be inapplicable in the treatment of chronic spinal cord injuries since hypothermia would not be expected to break down scar tissue already present at the site of SCI. If SCC were found to be effective, its use would be in the treatment of acute spinal cord injuries.

A recent article provided extensive discussion on the potential mechanisms for hypothermic protection for an SCI. These mechanisms of SCC included metabolic consequences, hemodynamic consequences, excitability, blood brain barrier aspects, calcium-dependent intracellular signaling, inflammation and edema, neuronal cell death, and global molecular changes [21]. Some of these characteristics may eventually prove to be beneficial in influencing a number of structural and functional problems that can develop within an injured spinal cord. A mechanism not mentioned, however, was the apparent failure of hypothermic absorptive capability, a mechanism that appears crucial in an acute SCI.

The article which originated the idea that SCC could lead to absorption of edema and blood began with an often referenced paper written in 1973 which claimed that SCC resulted in increased edema and blood absorption when applied after experimental SCI in cats [22]. This short paper (538 words) claimed that following an SCI, "hypothermia revealed a lack of penetration by hemorrhage and edema and gross preservation of normal cytoarchitecture." What was illustrated to demonstrate this reaction were two $1 \frac{1 / 2}{2}$ inch diagrammatic hand drawings with intermittent shaded areas that represented severe edema and hemorrhage with clear areas that were reported to be "protected by the hypothermia." This claim deserves careful consideration since traumatic edema and blood accumulation in the SC spreads by diffusion throughout the cross-sectional area of the SC; fluid absorption is not a localized phenomenon. Hypothermia can stabilize structures and solidify fluids-but it apparently cannot cause blood and fibrinogen absorption.

In an attempt to locate information as to whether SCC might have even limited ability to absorb edema and blood in an SCI, individuals in the fields of physics and engineering were consulted but no confirmation was found of a physical law that hypothermia could lead to the absorption of fluids. An exception was a report which stated that the external application of a $10^{\circ}$ temperature drop of bathing liquid surrounding single neurons produced approximately a $10 \%$ decrease in individual neuronal volume [23]. This small decrease in neuronal volume would not be expected to have an effect on absorptive activity within an injured spinal cord.

A strong argument has been made by proponents of SCC that an injured SC could be protected by hypothermia based on the reported protection of the spinal cord by SCC during the course of a surgical operation for thoraco-abdominal aneurysms [24]. What must be remembered, however, is that hypothermia was administered to the spinal cord during the operation at which time there was complete cessation of blood to the SC and no direct injury to the SC. This meant that in the absence of blood and fibrinogen in the uninjured spinal cord, it would be expected that hypothermia would be effective in protecting an SCI. However, in the presence of an actual injury to the SC in which blood and fibrinogen would be present, hypothermia would have little effect in absorbing blood and its constituents which is instrumental in the post-injury effects occurring in an SCI.

\section{Conclusion}

The long-term unfavorable neurological results that frequently occur following an SCI appear to be due to the scar formation that develops in an SCI. The presence of scar prevents the penetration of axons through the scar tissue, which limits the development of synaptic connections to the distal spinal cord. Fibrinogen is known to inhibit neurite growth and apparently acts as a key factor in the induction of the complex cascade that occurs in the production of spinal cord scar.

In an acute spinal cord injury, early absorption of fibrinogen is important in limiting the subsequent development of scar tissue. With a chronic SCI, however, absorption of blood is not a factor since scar formation is already present. In this situation, a pharmaceutical method in the future will have to be developed to absorb scar tissue. But presently a significant amount of scar in the SCI may have to be surgically excised and replaced with some sort of connection, such as an omental-collagen bridge, in order to connect the separated segments of the spinal cord. There appears at this time limited evidence that the use of stem cells, drugs, and spinal cord cooling will eventually be effective in absorbing blood and fibrinogen which are present in an acute SCI or in eliminating scar tissue in a chronically injured spinal cord. Let us hope that in the future newly developed methods will become effective in limiting the catastrophic effects of a serious SCI.

\section{References}

1. S. Ramon Y Cajal (1950) Degeneration and regeneration of the nervous system. Translated and edited by Raoul M. May, Hafner Publishing, New York 749-750.

2. FREEMAN LW (1961) Neuronal regeneration in the central nervous system of 
Citation: Goldsmith HS (2012) Omental Application in the Treatment of Spinal Cord Injuries: A Controlled Study is Warranted. J Trauma Treat 1:127. doi:10.4172/2167-1222.1000127

Page 6 of 6

man. Successful growth of intercostal-spinal nerve anastomosis and growth of intercostal nerve-spinal cord implant. J Neurosurg 18:417-22.

3. Nĕmecek S, Petr R, Suba P, Rozsival V, Mĕlka O (1977) Longitudinal extension of oedema in experimental spinal cord injury--evidence for two types of posttraumatic oedema. Acta Neurochir (Wien) 37: 7-16.

4. Schachtrup C, Lu P, Jones LL, Lee JK, Lu J, et al. (2007) Fibrinogen inhibits neurite outgrowth via beta 3 integrin-mediated phosphorylation of the EGF receptor. Proc Natl Acad Sci U S A 104: 11814-11819.

5. Goldsmith HS, Steward E, Duckett S (1985) Early application of pedicled omentum to the acutely traumatised spinal cord. Paraplegia 23: 100-112.

6. Goldsmith HS, De los Santos R, Beattie EJ Jr (1967) Relief of chronic lymphedema by omental transposition. Ann Surg 166: 573-585.

7. Levander B, Zwetnow NN (1978) Bulk flow of cerebrospinal fluid through a lumbo-omental pedicle graft in the dog. Acta Neurochir (Wien) 41: 147-155.

8. Shimada $Y$ (1995) Experimental study on effects of omental transposition in cats with spinal cord injury. No To Shinkei 47: 863-873.

9. Goldsmith HS (2010) Application of the omentum to the brain and spinal cord. In: Goldsmith HS (edtn). The Omentum-Basic Research and Clinical Applications. Cine-Med Publishing: Waterbury, CT USA.

10. Goldsmith HS (1986) Omental transposition to the brain and spinal cord. Surg Rounds 9: 22-23.

11. Goldsmith HS (2009) Treatment of acute spinal cord injury by omental transposition: a new approach. J Am Coll Surg 208:289-292.

12. Goldsmith HS (2002) Omental transposition to the spinal cord.In: Ostrander LE, Lee BY, Eds. The Spinal Cord Injured Patient.Demos Publisher, New York.

13. Goldsmith HS (2007) Can the standard treatment of acute spinal cord injury be improved? Perhaps the time has come. Neurol Res 29: 16-20.
14. Goldsmith HS, de la Torre JC (1992) Axonal regeneration after spinal cord transection and reconstruction. Brain Res 589: 217-224.

15. de la Torre JC, Goldsmith HS (1992) Supraspinal fiber outgrowth and apparent synaptic remodelling across transected-reconstructed feline spinal cord. Acta Neurochir (Wien) 114: 118-127.

16. de la Torre JC, Goldsmith HS (1994) Coerulospinal fiber regeneration in transected feline spinal cord. Brain Res Bull 35: 413-417.

17. Goldsmith HS, Fonseca A Jr, Porter J (2005) Spinal cord separation: MR evidence of healing after omentum-collagen reconstruction. Neurol Res 27 115-123.

18. de la Torre JC, Goldsmith HS (1990) Collagen-omental graft in experimental spinal cord transection. Acta Neurochir (Wien) 102: 152-63.

19. Guest JD, Dietrich WD (2005) Spinal cord ischemia and trauma. In: Tisherman SA, Sterz P, Therapeutic Hypothermia. Kluwer Academic Publishers, Boston.

20. Levi AD, Green BA, Wang MY, Dietrich WD, Brindle T, et al. (2009) Clinica application of modest hypothermia after spinal cord injury. J Neurotrauma 26 : 407-415.

21. Dietrich WD, Atkins CM, Bramlett HM (2009) Protection in animal models of brain and spinal cord injury with mild to moderate hypothermia. J Neurotrauma 26: 301-312.

22. Green BA, Khan T, Raimondi AJ (1973) Local hypothermia as treatment of experimentally induced spinal cord contusion: quantitative analysis of beneficient effect. Surg Forum 24: 436-438.

23. Austin G, Cushman A, Horn N (1972) Spinal Cord Edema. In: Austin G, The Spinal Cord: Basic Aspects and Surgical Considerations. Springfield, IL.

24. Cambria RP, Davison JK (2000) Regional hypothermia with epidural cooling for spinal cord protection during thoracoabdominal aneurysm repair. Semin Vasc Surg 13: 315-24 